
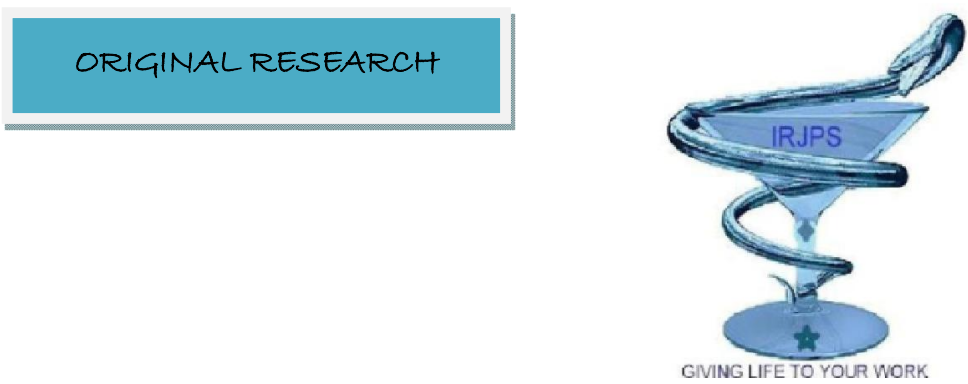

\title{
A STUDY ON PROPORTION OF COMORBIDITIES AND QUALITY OF LIFE IN PATIENTS WITH CHRONIC OBSTRUCTIVE PULMONARY DISEASE IN A TERTIARY CARE HOSPITAL
}

\author{
Arathy M.R ${ }^{1}$, Deepika $\mathbf{G}^{1}$, Hisana Thasneem K.A ${ }^{1}$, Soumya R.V ${ }^{1}$, Dr. P Ravindran ${ }^{2}$ \\ ${ }^{1}$ Department Of Pharmacy Practice, Sree Krishna College Of Pharmacy and Research Centre, Trivandrum, Kerala, \\ India \\ ${ }^{2}$ Cosmopolitan Hospital Pvt. Ltd, Pattom, Thiruvanathapuram
}

Submitted on: 29.11.16;

Revised on: 06.12.16;

Accepted on: 20.12.16

\begin{abstract}
:
Objective : A hospital based prospective observational study was done at a tertiary care hospital over a period of six month to analyse the clinically relevant co-morbidities, and quality of life in COPD patients.

Methods : The study included a total of 85 COPD patients who met the inclusion criteria and the proportion of comorbidities were assessed from the inpatient case records and Quality Of Life(QOL) was assessed using St. George Respiratory Questionnaire for COPD(SGRQ-C).

Result : The most common co-morbidities were Hypertension 55(64.7\%), Diabetes mellitus 32(37.6\%), Coronary Artery Disease $23(27.1 \%)$ and other heart disease $13(15.3 \%)$. The least common co-morbidities were Tuberculosis $2(2.4 \%)$, Osteoporosis $1(1.2 \%)$ and DVT $1(1.2 \%)$ etc. In quality of life, the mean symptom score was found to be 62.1 with standard deviation of 19.3, mean activity score was found to be 71.7 with the standard deviation of 19.7 and mean impact score was found to be 52.6 with the standard deviation of 17.8 . The mean overall quality of life score was found to be 59.3 with the standard deviation of 15.9 .

Conclusion : It was observed that the clinically relevant co-morbid condition often coexisted in exacerbated COPD patients and had a moderate quality of life. The diagnosis and management of co-morbidities is an important challenge for the COPD patient as it is not always easy to diagnose the coexisting illness. Thus, systematic research of co-morbidities seems to be of interest. Thereby improving the medication adherence and addressing the comorbid conditions we can improve the quality of life as well as reduce the mortality and morbidity in COPD patients.
\end{abstract}

KEYWORDS: COPD, Quality Of Life, Co-morbidities, SGRQ-C

Corresponding author: Soumya R.V Email id:tosoumyarv@gmail.com
Indian Research Journal of Pharmacy and Science; 11(2016) 842-848; Journal Home Page: https://www.irjps.in 


\section{INTRODUCTION:}

Chronic Obstructive Pulmonary Disease (COPD) is a leading cause of morbidity and mortality worldwide, especially among smokers over 40 years of age .WHO estimates COPD is the fifth most common worldwide cause of death. Chronic obstructive pulmonary disease(COPD) is a term which refers to a large group of lung diseases characterised by limitation of air flow that interferes with normal breathing ${ }^{1}$.

According to the definition of GOLD guidelines "COPD is a common preventable and treatable disease, characterised by air flow limitation that is usually progressive and associated with an enhanced chronic inflammatory response in the airways and lungs to noxious particles or gases". Emphysema and chronic bronchitis are the most important conditions that compose COPD and they frequently coexist ${ }^{2}$.

\section{COPD and Its Co-morbidities}

A comorbidity is usually defined as a disease coexisting with disease of interest. An important factor in both prognosis and functional capabilities of COPD patients is the role of comorbid disease ${ }^{3}$. It is significant because comorbidities in COPD is associated with poorer outcomes. Studies have shown that variety of chronic conditions associated with COPD include hypertension, diabetes mellitus, coronary artery disease(CAD), depression , malignancy, osteoporosis etc. Majority of patients with COPD have at least one co-morbidities. Comorbidities markedly affect the health outcomes in $\mathrm{COPD}^{4}$.Chronic diseases, including cardio vascular diseases, cancer and metabolic syndrome are increasingly associated with COPD result in substantial social and economic burden ${ }^{5}$. Most common chronic disease associated with COPD is congestive heart failure related with severity of air flow obstruction. COPD patients often have one or more component of metabolic syndrome either hypertension or diabetes mellitus and osteoporosis ${ }^{6}$. These co-morbidities were reported to increase the risk of mortality and

hospitalisations in COPD patients and they exponentially increase the cost of treatment.

\section{Quality Of Life}

Quality of Life (QOL) is the general well being of individuals and societies, outlining negative and positive features of life. It observes life satisfaction, including everything from physical health, family, education, employment, wealth, religious beliefs, finance and the environment. Quality of life in COPD patients can be measured by generic or disease specific instruments. Health related quality of life( HRQOL) measurement using validated questionnaires provides a standardised measure for assessing the impact of disease on patient's daily lives, activity and well being. Generic questionnaires are designed to assess health status irrespective of underlying disease, while disease specific questionnaires tend to be more sensitive to change. Among disease specific instruments, St. George Respiratory

Questionnaire has been considered the gold standard for assessment of HRQOL in patients with COPD. Large epidemiological studies have used SGRQ was impaired in COPD subjects and was independently associated with hospital readmission and mortality. Comorbidities are known to affect the morbidity, mortality and healthy related quality of life . $^{3}$

The present study evaluates the proportions of comorbidties and the Health Related Quality Of Life in COPD patients in a tertiary care hospital. When present, clinically relevant comorbidities significantly affects the quality of life in COPD patients. Management of co-morbid conditions is very important in case of a chronic progressive disease like COPD. This study highlights the need for addressing the co-morbid condition and would be further effective in improving the quality of life of patients.

\section{MATERIALS AND METHODS:}

This study was conducted in the Pulmonary and Critical care Department of Cosmopolitan hospital, Thiruvananthapuram for a period of six months after getting approval from Institutional Ethics Committee. A total 85 patients were included after obtaining the consent. The study included all the inpatients of both gender above 40 years of age diagnosed COPD. The patients aged below 40 years and who were critically ill and those whom death occur within one week of hospital admission were excluded.The case records were reviewed and the details were collected during the period of hospital stay and a proforma was designed to assess the demographics, symptoms, comorbidities, past medication history, diagnosis and treatment regimen of the patients.

The relevant data was analysed and the proportion of co-morbidities were determined from the patient's case records. Quality of life was assessed using St. George respiratory questionnaire for COPD (SGRQ C) which is a self administered questionnaire and the scoring was done accordingly into three categories ie symptom score, activity score, impact score. Then the total score was calculated. The 
descriptive statistical analysis such as mean, standard deviation and percentage was used to describe variables under study.

\section{RESULTS:}

A total of 85 patients met the study criteria. Out of the 85 patients $47(55.3 \%)$ patients were male and 38 (44.7\%) were females (fig 1)

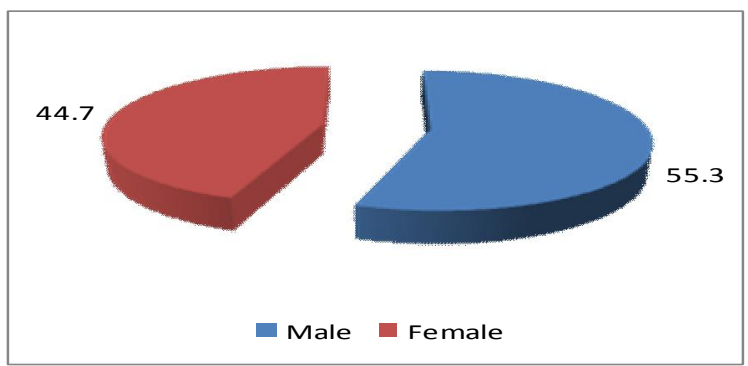

Figure 1: Percentage distribution of the sample according to sex

Fourty eight patients, $(56.5 \%)$ found to be over 70 years age. Twenty one patients $(49 \%)$ belong to age group of 61-70 years. Only eight patients $(9.4 \%)$ belong to the age group of 51-60 years of age ( fig 2).

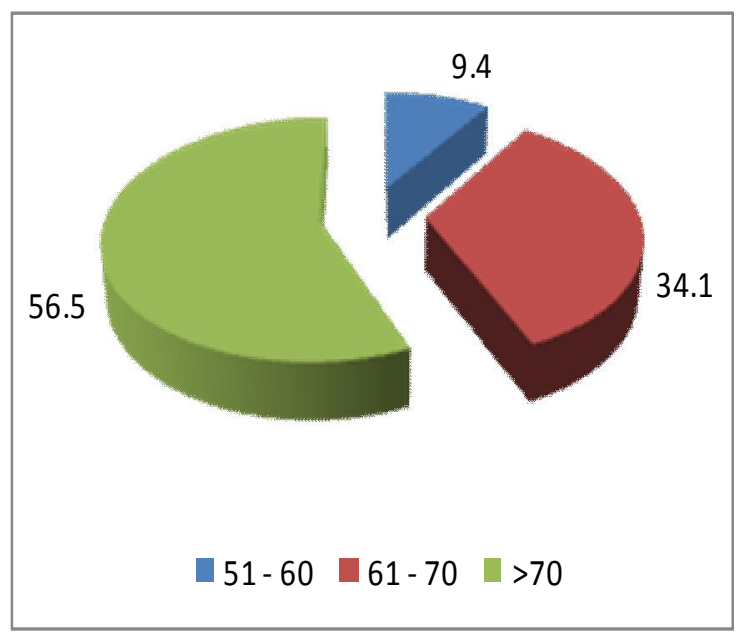

Figure 2: Percentage distribution of the sample according to age

The most common co-morbidities were Hypertension 55(64.7\%), Diabetes mellitus 32(37.6\%), Coronary Artery Disease 23(27.1\%) and other heart disease 13 $(15.3 \%)$. The least common comorbdities were TB 2 $(2.4 \%)$,Osteoporosis $1(1.2 \%)$ and DVT 1 (1.2\%) (fig $3)$.

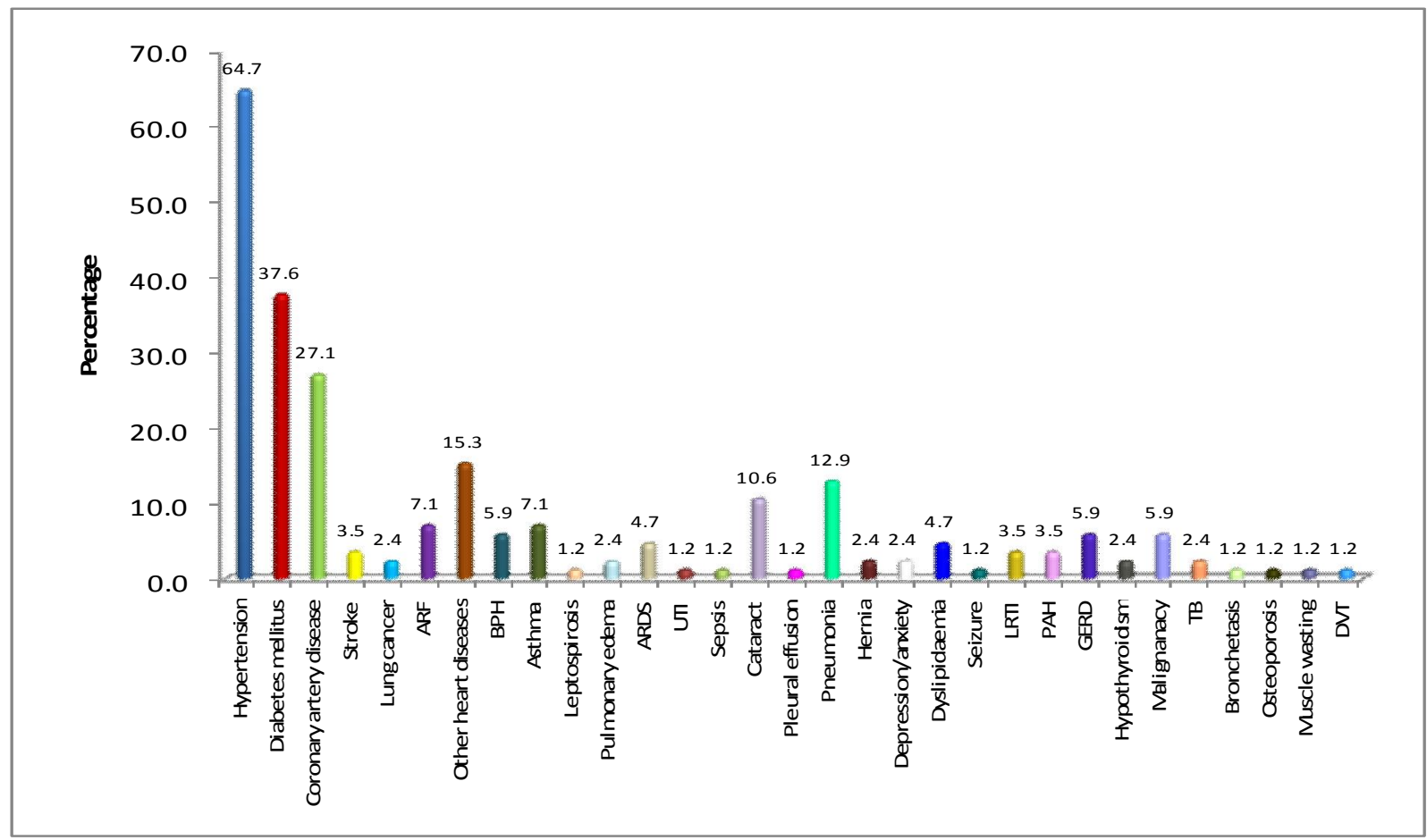

Figure 3: Percentage distribution of the sample according to co morbidities 


\section{Health Related Quality of Life in COPD patients}

In quality of life, the mean symptom score was found to be 62.1 with standard deviation of 19.3 (fig 4, Table 1) mean activity score was found to be 71.7 with the standard deviation of 19.7 (fig 5, Table 2)and mean impact score was found to be 52.6 with the standard deviation of 17.8 (fig 6 , table 3 ) .The mean overall quality of life score was found to be 59.3 with the standard deviation of 15.9 (fig 7, table 4)

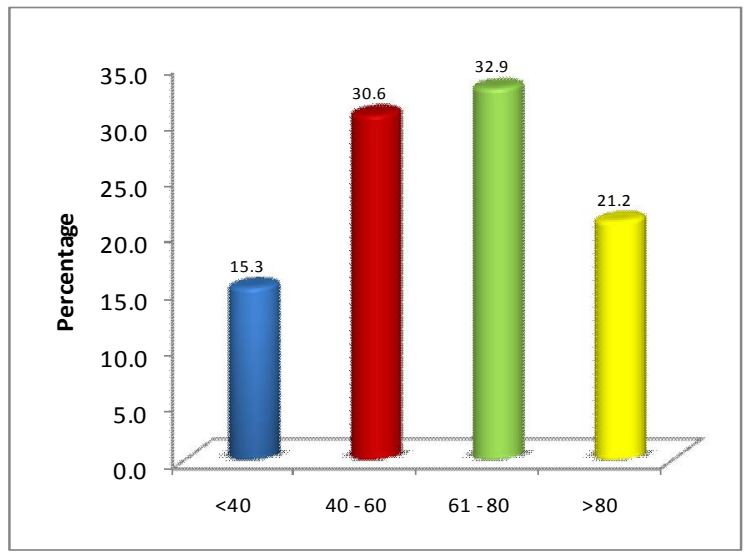

Figure 4:Percentage distribution of the sample according to symptom

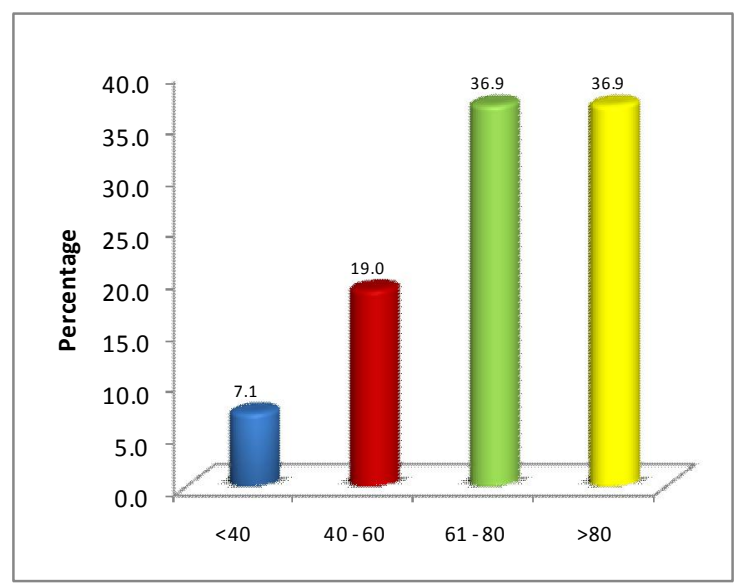

Figure 5: Percentage distribution of the sample according to activity

\begin{tabular}{|c|c|c|}
\hline Activity & Count & Percent \\
\hline$<40$ & 6 & 7.1 \\
\hline $40-60$ & 16 & 19.0 \\
\hline $61-80$ & 31 & 36.9 \\
\hline$>80$ & 31 & 36.9 \\
\hline Mean \pm SD & \multicolumn{2}{|c|}{$71.7 \pm 19.7$} \\
\hline
\end{tabular}

Table 2: Percentage distribution of the sample according to activity

\begin{tabular}{|c|c|c|}
\hline Symptoms & Count & Percent \\
\hline$<40$ & 13 & 15.3 \\
\hline $40-60$ & 26 & 30.6 \\
\hline $61-80$ & 28 & 32.9 \\
\hline$>80$ & 18 & 21.2 \\
\hline Mean \pm SD & \multicolumn{2}{|c|}{$62.1 \pm 19.3$} \\
\hline
\end{tabular}

Table 1: Percentage distribution of the sample according to symptoms

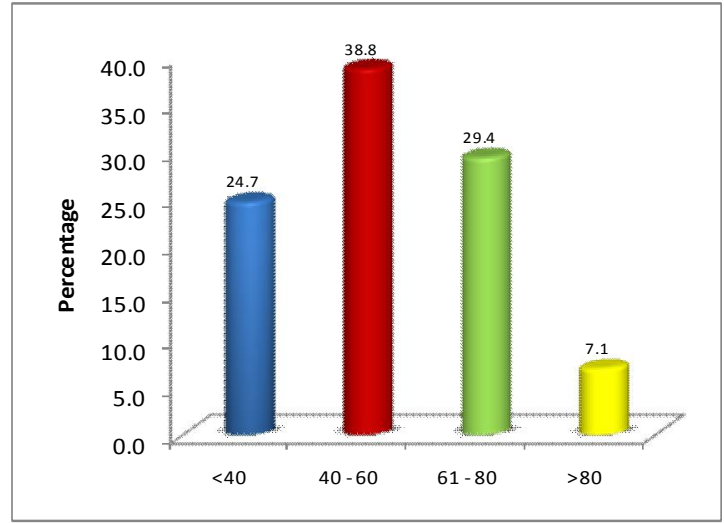

Figure 6: Percentage distribution of the sample according to impact 


\begin{tabular}{|c|c|c|}
\hline Impact & Count & Percent \\
\hline$<40$ & 21 & 24.7 \\
\hline $40-60$ & 33 & 38.8 \\
\hline $61-80$ & 25 & 29.4 \\
\hline$>80$ & 6 & 7.1 \\
\hline $\begin{array}{c}\text { Mean } \pm \\
\text { SD }\end{array}$ & \multicolumn{2}{|c|}{$52.6 \pm 17.8$} \\
\hline
\end{tabular}

Table 3: Percentage distribution of the sample according to impact

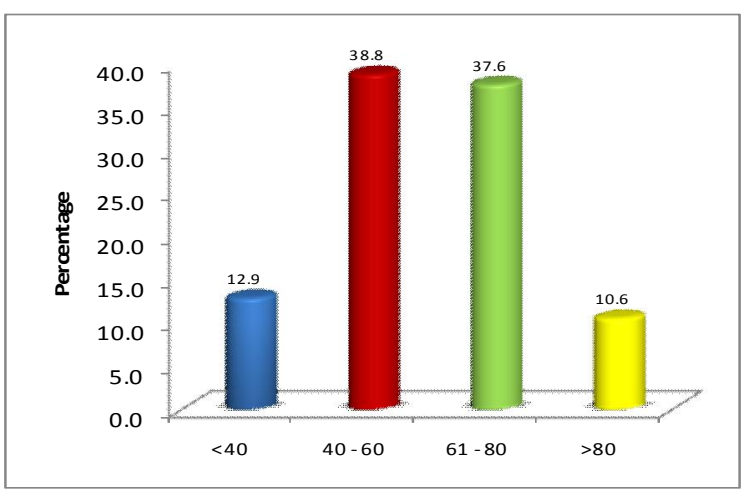

Figure 7: Percentage distribution of the sample according to overall quality of life

\begin{tabular}{|c|c|c|}
\hline $\begin{array}{c}\text { Overall quality } \\
\text { of life }\end{array}$ & Count & Percent \\
\hline$<40$ & 11 & 12.9 \\
\hline $40-60$ & 33 & 38.8 \\
\hline $61-80$ & 32 & 37.6 \\
\hline$>80$ & 9 & 10.6 \\
\hline Mean \pm SD & \multicolumn{2}{|c|}{$59.3 \pm 15.9$} \\
\hline
\end{tabular}

Table 4: Percentage distribution of the sample according to overall quality of life

\section{DISCUSSION:}

The present study highlights the proportion of comorbidities and quality of life in COPD patients. From the study of 85 patients during a period of six month in a tertiary care hospital, it was found that the most commonly affected age group in this study was above 70 years [fig:2] and similar results were found by Elisabeth Stahl et $\mathrm{al}^{7}$, who found highest incidence at the mean age of 64 . The result of the study done by Gregory B Diette et al $^{8}$ also states that the mean age was 66 years. In our study the occurrence of COPD were found more in males (55.3\%) than in females (44.7\%) [fig:5] is mainly due to the exposure to environmental risk factor cigarette smoke . Same result were found in study by Pat $G$ Camp et $a l^{9}$ i.e, the prevalence is more in males .

From our study the most relevant co-morbidities were Hypertension (64.7\%), followed by Diabetes mellitus( $37.6 \%$ ), and coronary artery disease $(27.1 \%)$. The least common co-morbidities were TB (2.4\%) , Osteoporosis (1.2\%) and DVT (1.2\%). According to study by Catherina Anecchino et $a l^{10} 98 \%$ of patients received at least one prescription of non respiratory drugs for chronic specific comorbidities(cardiovascular disease, diabetes and depression) . $68.4 \%$ suffered from at least one of this condition with cardiovascular disease (64.4\%), diabetes( $12.4 \%)$ and depression (8\%) Systemic inflammation observed in COPD is key determinant for pulmonary and systemic endothelial dysfunction and coagulopathy which lead to most of the major cardio vascular co-morbidities and venous thromboembolism. Smoking is a risk factor that coexist for both COPD and CAD.

In this study, the Health related quality of life (HRQOL) in COPD exacerbated patients was assessed using St. George Respiratory questionnaire for $\mathrm{COPD}(\mathrm{SGRQ}-\mathrm{C})$. From this symptom, activity and impact score was determined to determine the over all quality of life(QOL). The mean symptom score was found to be 62.1 with standard deviation of 19.3 , mean activity score was found to be 71.7 with the standard deviation of 19.7 and mean impact score was found to be 52.6 with the standard deviation of 17.8. The mean overall quality of life score was found to be 59.3 with the standard deviation of 15.9. Here the patients had shown a poor to moderate health related quality of life. Yavuz Havlucu e.tal study shows that the mean SGRQ scores of subscale activity, symptom, impact and total were $58.6 \pm 17.2$, $57.2 \pm 13.9,42.7 \pm 16.6$ and $48.9 \pm 15.4$ respectively and also found that number of exacerbations per year showed an increase with disease severity. In addition 
current smoking caused an increase in SGRQ scores by increasing exacerbation frequency, although it did not directly cause quality of life deterioration ${ }^{11}$.

The main limitations of this study include its short duration of six months. In that duration we were only able to include 85 patients in which most of them were exacerbated inpatients. As it is not possible to assess the quality of life accurately in case of exacerbated patients. We assessed the co-morbidities using patient's medical records, so we got only the co-morbidities that were physician diagnosed. Thus could not analyse whether the co-morbidities were present throughout the disease course and was not able to detect an association with the course of COPD.

\section{REFERENCE:}

1. Georgios Hillas,Fotis Perlikos,Ioanna Tsiligianni,Nikolaos Tzanakis " Managing co morbidities in COPD" International Journal of COPD 2015:10 95-109

2. Global Initiative for Chronic Obstructive Lung Disease. Global strategy for the diagnosis, management and prevention of chronic obstructive pulmonary disease. Updated 2015.

3. Koskela J, Kilpeläinen M, Kupiainen $\mathrm{H}$, Mazur W, Sintonen H, Boezen M, Lindqvist A, Postma D, and Laitinen $\mathrm{T}$ "Co-morbidities are the key nominators of the health related quality of life in mild and moderate COPD." BMC Pulm Med. 2014;14(1):102.

4. Divo M, Cote C, de Torres J, Casanova C, Marin J, Pinto-plata V, Zulueta J, Cabrera C, Zagaceta J, Hunninghake G, Celli B "Comorbidities and risk of mortality in patients with chronic obstructive pulmonary disease". Am J Respir Crit Care Med. 2012;186(2):155-161

5. Wissam M. Chatila, Byron M. Thomashow, Omar A. Minai, Gerard J. Criner, and Barry J. Make"Comorbidities in Chronic Obstructive Pulmonary Disease" Proc Am Thorac Soc Vol 5. pp 549-555, 2008,Proc Am Thorac Soc Vol 5. pp 549555,2008

\section{CONCLUSION:}

The present study was conducted to assess the clinically relevant co-morbidities and quality of life in COPD patients..It was observed that the clinically relevant co-morbid condition often coexisted in exacerbated COPD patients along with a moderate quality of life. These co-morbidities have a greater negative impact in COPD patients in terms of quality of life, exacerbation and mortality. Thus, diagnosis and management of co-morbidities is an important challenge for the COPD patient as it is not always easy to diagnose the coexisting illness. Indeed, these can be asymptomatic or the symptoms may not be specific in COPD patient. Thus, systematic research of co-morbidities seems to be of interest. Thereby improving the medication adherence and addressing the comorbid conditions we can improve the quality of life as well as reduce the mortality and morbidity in COPD patients.

6. Miranda Caroline Smith, Jeremy $\mathrm{P}$ Wrobel, " Epidemiology and clinical impact of major

comorbidities in patients with COPD" International Journal of COPD 2014:9 871-888

7. Elisabeth Ståhl, Lindberg A, Jansson SA, Ronmark E, Svensson K, Andersson F, Lofdahl $\mathrm{CG}$, Lundvack B "Health-related quality of life is related to COPD disease severity”Health and Quality of Life Outcomes 2005, 3:56.

8. Gregory B Diette, Anand Adalal, Anna O D’Souza, Orsolya Elunacsek, Saurabh $\mathrm{P}$ Nagar "Treatment patterns of chronic obstructive pulmonary disease in employed adults in the United States", International Journal of COPD 2015:10 415-422.

9. Pat G Camp, Denis E.O' Donnel and Dirkje S.Postma "Chronic obstructive pulmonary disease in men and women", Proceedings of American Thoracic Scociety, Vol 6, No. ^ (2009) pp 535-538.

10. Caterina Anecchino, Elisa Rossi, Caterina Fanizza, Marisa De Rosa, Gianni Tognoni, Marilena Romero, "Prevalence of chronic obstructive pulmonary disease and pattern of comorbidities in a general population" International Journal of COPD 2007:2(4) 567-574.

11. Yavuz Havlucu, Pinar clik, Gonul Dinc, Aysin Sakar, Arzu Yorgancioglu "Assessment of 
Quality of Life in Chronic Obsturctive Pulmonary Disease"Turkish respiratory Journal,2005 ,vol.6 ,issue 2, pp 78-83

\section{ABBREVIATIONS:}

COPD - Chronic Obstructive Pulmonary Disease QOL - Quality Of Life

SGRQ-C - St George Respiratory Questionnaire for COPD

DVT - Deep Vein Thrombosis

WHO - World Health Organization

GOLD - Global Initiative for Obstructive Lung
Disease

CAD - Coronary Artery Disease

HRQOL - Health Related Quality Of Life

TB - Tuberculosis

ARF - Acute Renal Failure

BPH - Benign Prostatic Hyperplasia

ARDS - Acute Respiratory Distress Syndrome

UTI - Urinary Tract Infection

LRTI - Lower Respiratory Tract Infection

PAH - Pulmonary Arterial Hypertension

GERD - Gastro Esophageal Reflux Disease 